\title{
Immunizing Mice using Recombinant Truncated p72 Protein of African Swine Fever Virus and Establishment of an Indirect ELISA
}

\author{
Jinliang Wang ${ }^{1 * \dagger}$, Weiqin Meng ${ }^{1 \dagger}$, Jinlong Chen ${ }^{2}$, Lin Dong ${ }^{1}$, Xinyou Yu ${ }^{2}$ and Zhiqiang Shen ${ }^{1,2^{*}}$ \\ ${ }^{1}$ Key Laboratory of Preventive Veterinary Medicine and Animal Biotechnology, Shandong Binzhou Animal Science and \\ Veterinary Medicine Academy, Binzhou, 256600, China \\ ${ }^{2}$ Shandong Lvdu Bio-Sciences and Technology Co., Ltd., Binzhou, 256600, China \\ *For correspondence: WJL478@163.com; bzshenzq@163.com \\ † Contributed equally to this work and are co-first authors \\ Received 11 November 2020; Accepted 19 November 2020; Published 25 January 2021
}

\begin{abstract}
African swine fever (ASF) is a serious infectious pestilence characterized by bleeding in domestic pigs. Therefore, it is necessary to develop effective methods to diagnose this virus, serological detection of specific antibodies against ASFV infection is important for successful clinical diagnosis. In this study, E. coli was used to express the truncated P72 (tP72) gene cloned into the prokaryotic expression vector pET28a (+). Rosetta (DE3). An indirect ELISA assay which against African swine fever virus (ASFV) was established by using purification of recombinant tP72 protein as coated material for detection antibodies. Most effective in exhibiting positive result was observed when the coated material at a concentration of 3.625 $\mu \mathrm{g} / \mathrm{mL}$, serum was diluted to 1:160 and the concentration of HRP-conjugated secondary antibody was 1:2000. Our results showed that the method displayed an excellent specificity (100\%) and better sensitivity (1:1600) during serological test based on the criterion of an average value plus three standard deviations. (C) 2021 Friends Science Publishers
\end{abstract}

Keywords: African swine fever virus; Truncated p72 protein; Prokaryotic expression; Mice; Indirect ELISA

\section{Introduction}

African swine fever (ASF) is a serious infectious pestilence that is caused by the African swine fever virus (ASFV) infection. ASFV infection domestic pigs, had caused huge economic loss. So far there are no effective commercial vaccines to against the ASF infection (Costard et al. 2013; Detray 1963; Galindo and Alonso 2017). ASFV possesses a double stranded DNA genome. Moreover, the C-terminal region of p72 (B646L) has been traditionally used for the genotyping of ASFV isolates, more than $20 \mathrm{ASFV}$ genotypes have been identified. The p72 is one of the most immunogenic ASFV protein and constitutes about $32 \%$ of the total virus mass, and is an important target for test and vaccine development (Mur et al. 2016; Achenbach et al. 2017; Mulumba-Mfumu et al. 2017).

In the $1920 \mathrm{~s}$, for the first time ASF was discovered in Kenya and spread to Portugal, Cuba, Brazil, Dominican Republic and Haiti in 1957. In addition, in 2007, ASF was led into Georgia, then drawn into Russia and Ukraine, along with diffused to European Union countries such as Latvia, Poland, Estonia and Lithuania in 2017 (Montgomery 1921; Sanchez-Vizcaino et al. 2012; Achenbach et al. 2017). A new outbreak of ASF has affected domestic pigs around
Liaoning province of China since August 2018. The disease of ASF has caused severe economic losses, hence, there is an urgent need to develop efficacious vaccines to control the spread of the pestilence (Carolina et al. 2013; Gallardo et al. 2013; Sastre et al. 2016a, b). As the major structural protein of ASFV, the p72 is commonly used as the antigen for the purpose of serologic detection. Based on previous studies, a truncated p72 recombinant protein was obtained by $E$. coli expression system. An indirect ELISA method with the purified p72 protein as coating material was developed to identify the ASFV-specific antibodies. This method can be used for epidemiological investigation of ASF.

\section{Materials and Methods}

\section{Bacterial strain, vector, Enzymes and reagents}

Plasmid reference material of $\mathrm{B} 646 \mathrm{~L}$ gene of ASFV (GBW(E) 091034) was provided by Shenzhen kangbaide Biotechnology Co., Ltd. E. coli DH5 $\alpha$, E. coli Rossetta (DE3) and pET28a (+) vector were purchased from Sangon Biotech (Shanghai) Co., Ltd. Healthy mice ( 22g), 6-weekold, were bought from the Binzhou medical university (Binzhou, China). BamH I, Xho I, T4 DNA ligase, rTaq

To cite this paper: Wang J, W Meng, J Chen, L Dong, X Yu, Z Shen (2021). Immunizing mice using recombinant truncated p72 protein of African swine fever virus and establishment of an indirect ELISA. Intl J Agric Biol 25:609-614 
DNA polymerase, and low molecular weight protein marker were provided by Jinan chengshen Co., Ltd (Jinan, China). HRP conjugated goat anti-rabbit IgG was purchased from Solarbio (Beijing, China). Microtiter plates (96-well) and Ni-NTA agarose were provided by Qingdao haosai Co. Ltd. (Qingdao, China).

\section{Cloning of truncated p72 gene (tP72)}

Based on the p72 gene sequences of ASFV (GenBank accession No. MK128995.1), one pair of p72 gene primers, forward primer 5'CGCGGATCCGCATCAGGAGGAGCTTTT-3' nucleotides downstream of the ATG start site in the p72 gene) and reversed primer 5'CCGCTCGAGAACCTGCTGTTTGGATATT.

G-3' (873 nucleotides downstream of the ATG start site in the p72 gene) containing $\operatorname{BamH~I}$ and Xho I restriction sites at the $5^{\prime}$ terminus (underlined), respectively, were designed with Primer 5.0 software.

The $25 \mu \mathrm{L}$ PCR reaction system consisted of $12.5 \mu \mathrm{L}$ of PCR reaction buffer mixture (2-fold), $1.5 \mu \mathrm{L}$ of each primer $(10 \mu \mathrm{M}), 2 \mu \mathrm{L}$ of DNA template (plasmid reference material of $\mathrm{B} 646 \mathrm{~L}$ gene) and $7.5 \mu \mathrm{L}$ of ddH2O. The reaction procedure of PCR was: $94^{\circ} \mathrm{C}$ denaturation for $3 \mathrm{~min} ; 35$ cycles of $94^{\circ} \mathrm{C}$ for $30 \mathrm{~s}, 30 \mathrm{~s}$ at $52^{\circ} \mathrm{C}$ and $72^{\circ} \mathrm{C}$ for $30 \mathrm{~s}$; then performed at $72^{\circ} \mathrm{C}$ for 6 min for PCR final extension. PCR amplification products were detected using $1.5 \%$ agarose gel nucleic acid electrophoresis. The amplified target sequence was 870 bp in length. The tP72 gene fragment was cloned into pET28a (+) vector at the BamH I and Xho I sites (Fig. 1) and was checked through nucleotide sequencing (data not shown).

\section{Expression and purification of tP72 truncated protein}

The amplified tP72 gene was inserted into the BamHI/ Xho I site of prokaryotic expression vector pET28a(+) using standard molecular techniques (Carson et al. 2012), resulting in a recombinant plasmid designated as pET28a(+)/ tP72. E. coli strain Rossetta (DE3) were transformed with pET28a $(+) /$ tP72 plasmid and cultured in LB medium (ratio of 1:100) containing $50 \mathrm{mg} / \mathrm{mL}$ kanamycin. Cells were induced with $1 \mathrm{~m} M$ of IPTG (isopropyl-b-dthiogalactopyranoside) for $4 \mathrm{~h}$ at $37^{\circ} \mathrm{C}, 220$ $\mathrm{rpm}$. The cells were obtained by centrifugation for $10 \mathrm{~min}$ at $4^{\circ} \mathrm{C}, 3500 \mathrm{rpm}$, and resuspended in a propriate amount of PBS (pH7.4) buffer and sonicated. The protein was further purified with AKTA purifier 100. Sequentially, the purified tP72 protein was detected by protein electrophoresis and Western blotting.

\section{Mouse immunization}

Four female Kunming mice (about six weeks old) were immunized with purified tP72 antigen (60 $\mu \mathrm{g} / \mathrm{mouse})$ mixed with 3 times volume of oil adjuvant and the sera were collected every 7 days from the day post immunization to 42 days. At the same time, three mice were immunized with purified vector Tag protein and used as control.

\section{Establishment of an indirect ELISA}

An indirect ELISA protocol was designed according to the references (Crowther and Walker 2009; Bu et al. 2015), briefly, polystyrene microtiter paltes were coated $(100 \mu \mathrm{L}$ /well) with tP72 protein $(14500 \mu \mathrm{g} / \mathrm{mL})$ diluted with $0.05 M$ carbonate buffer $(\mathrm{pH}=9.0)$ and incubated at $2-8^{\circ} \mathrm{C}$ for $6 \mathrm{~h}$. After four 3-min rinses with $0.01 M$ PBS (phosphatebuffered saline, $\mathrm{pH}=7.4$ ) containing $0.05 \%$ Tween-20 (PBST), the 96-well plate was filled with $200 \mu \mathrm{L}$ of blocking solution ( $5 \%$ dry milk in PBST) at $37^{\circ} \mathrm{C}$ for $2 \mathrm{~h}$, and discarded the blocking solution, and then added $100 \mu \mathrm{L}$ of two-fold dilutions of the positive or negative sera (the dilution ratio is $1: 20,1: 40,1: 80,1: 160$ and 1:320, respectively) at $37^{\circ} \mathrm{C}$ for $30 \mathrm{~min}$, followed by four times with PBST. Secondary antibody-peroxidase conjugate was added to each well $(100 \mu \mathrm{L}$ at 1:2,000 dilution in $0.01 \mathrm{M}$ PBS) and the microtiter plates were incubated at $37^{\circ} \mathrm{C}$ for $30 \mathrm{~min}$. The washing step was repeated and finally, $100 \mu \mathrm{L}$ of Ultra TMB was added to the holes one by one and the microtiter plates were placed in the dark for $10 \mathrm{~min}$ at room temperature. An equal amount of stopping solution (1 M $\mathrm{HCl}$ ) was added and the optical density (OD) was determined at $450 \mathrm{~nm}$ wavelength using a plate reader. The optimal concentrations of coating tP72 protein and serum dilution were determined based on the ratio of reading values of positive and negative serum at OD450 nm wavelength $(\mathrm{P} / \mathrm{N})>2$.

Statistical analysis was performed to calculate the mean value $(\mathrm{X})$ and standard deviation (SD) of the OD450nm values of 50 serum samples. The threshold value was determined as $\mathrm{X}+3 \mathrm{SD}$. The cross reaction between the positive serum of Classical swine fever virus (CSFV), Porcine circovirus type 2 (PCV2), Porcine parvovirus (PPV), Japanese encephalitis virus (JEV), Porcine Epidemic Diarrhea Virus (PEDV), Porcine reproductive and respiratory syndrome (PRRSV) and Transmissible gastroenteritis virus (TGEV) was investigated using ELISA to analyze the specificity of the antigen. To validate the sensitivity of the tP72-based indirect ELISA, two-folddiluted positive serum starting at 1:100 was evaluated by indirect ELISA.

\section{Results}

\section{Construction of recombinant expression vector}

The target gene of tP72 was successfully ligated into the pET28a (+) vector, an expected band size of 870 base pairs (bp) was observed after digestion by double enzymes (BamH I and Xho I) when electrophoresed in 1\% agarose gel (Fig. 1). 


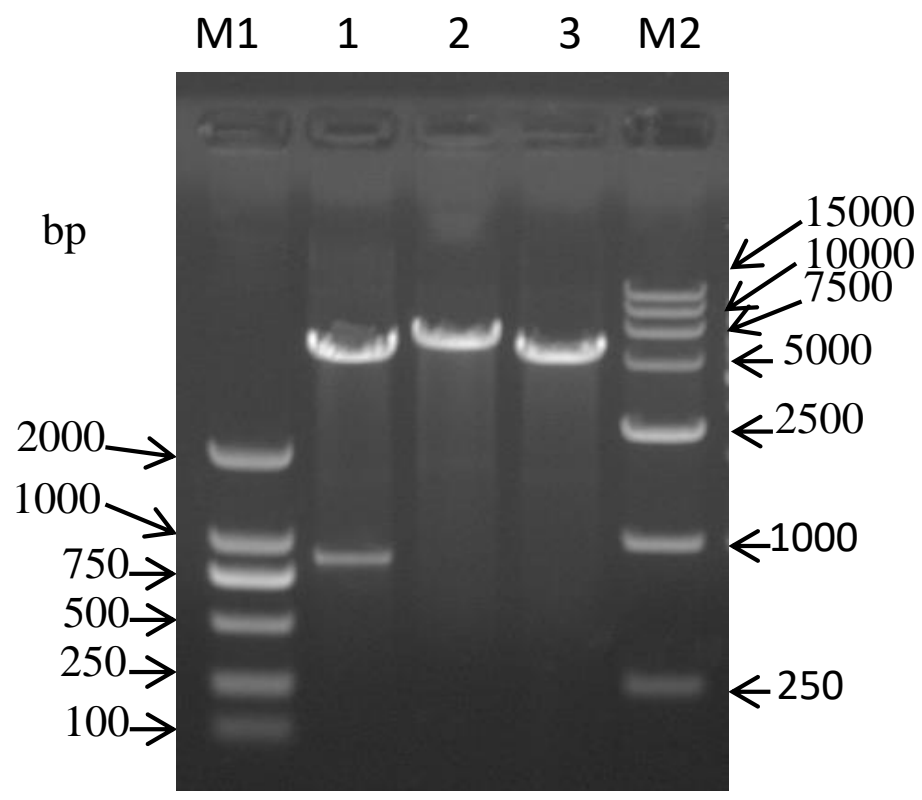

Fig. 1: Identification of pET28a (+)/tP72 vector

The lane M1 shows the DNA marker; lane 1 is the verification of pET28a (+)/tP72 vector which was cleaved by Xho I and BamH I; lane 2 is the verification of pET28a (+)/tP72 vector which was cleaved by Xho I; lane 3 is the cleaved pET28a (+) vector by Xho I and BamH I; the lane M2 is DL15000 DNA marker

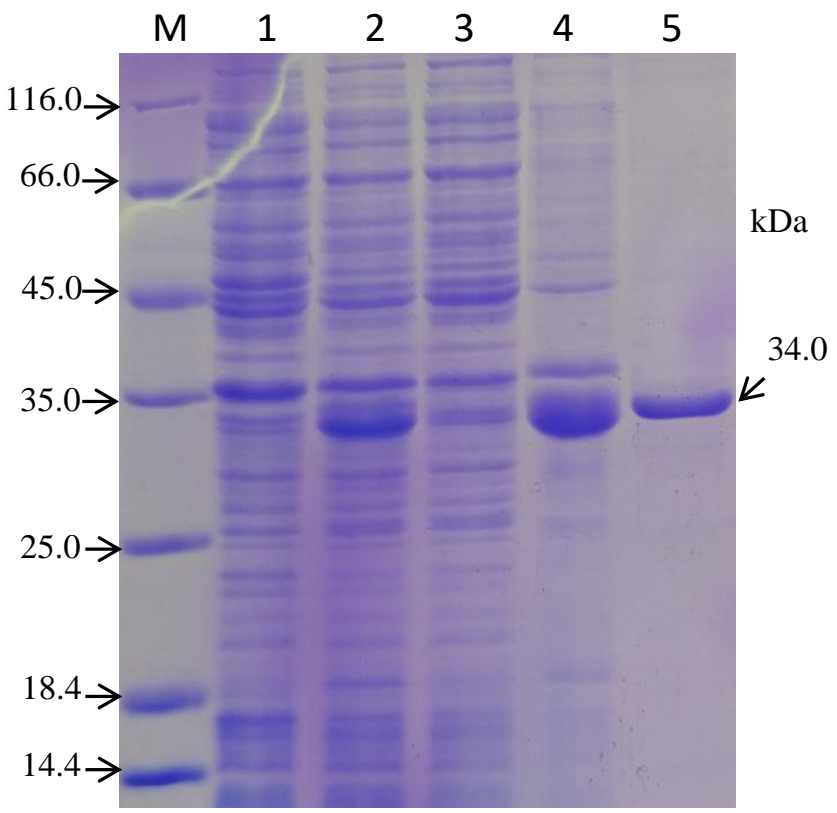

Fig. 2: Expression and purification of ASFV tP72 protein

Lane M is the protein maker; Lane 1, Expression of Rossetta/pET28a (+); lane 2, Expression of Rossetta/pET28a (+)/tP72; Lane 3 is the protein products in the supernatant of the bacterial lysate; Lane 4 is the protein products in the sediment of the bacterial lysate; Lane 5 shows the purified tP72 protein

\section{Expression and purification of tP72 truncated protein}

E. coli Rossetta (DE3) transformed with pET28a (+)/tP72 were induced with $1 \mathrm{mM}$ IPTG for $4 \mathrm{~h}$ at $37^{\circ} \mathrm{C}$ and harvested and sonicated as previously descried. The proteins were analyzed using SDS-PAGE (Fig. 2). The main part of tP72 protein was presented in the insoluble inclusion body.
The purified tP72 protein had the expected molecular size weight ( $34 \mathrm{kDa})$, containing the His-tag fusion peptide with a mass of about $5 \mathrm{kDa}$. Western blot assays confirmed that the expressed tP72 had specific reaction with anti His-tag mouse monoclonal antibody and anti ASFV positive serum (Fig. 3). The concentration of purified tP72 protein was 1.45 $\mathrm{mg} / \mathrm{mL}$ by BCA protein detection kit. 
Compared with the control group, levels of the IgG antibody of the mouse serum gradually increased from 7 to 14 days after tP72 protein immunization. The titer of the antibody in the serum rose to the highest level on 21d (increasing to the peak of 1:1600). At 42 days, the antibody in the serum was still positive (Fig. 4). Experimental data indicated that the $\mathrm{tP} 72$ has the potential to be used as a coated material to measure the antibody titer in the peripheral serum using indirect ELISA method.

\section{Development of an indirect ELISA using tP72}

Optimization conditions of the indirect ELISA were determined according to the square matrix titration test, the optimum conditions of ELISA were $3.625 \mu \mathrm{g} / \mathrm{mL}$ for the coated tP72 protein, namely $0.3625 \mu \mathrm{g}$ per hole and a $1: 160$ dilution of mouse serum, and a 1:2000 of secondary antibody-peroxidase conjugate, and the optimal reaction time was $30 \mathrm{~min}$. The critical value was determined according to the mean $\mathrm{OD}_{450 \mathrm{~nm}}$ value plus $3 \mathrm{SD}$ from 50 negative serum samples from mice using optimum conditions. The mean value of the OD450nm values of 50 samples was 0.215 and the SD value was 0.018 . Therefore, serum with OD450 $\mathrm{nm} \geq 0.269$ would be positive, when the difference between the OD450nm values of the standard positive serum and the negative serum was higher than 0.05 , $\mathrm{P} / \mathrm{N} \geq 2.0$.

\section{Detection for cross-reactivity of antibodies against other common porcine viruses}

In this study, mouse anti sera was used to investigated the Cross-reactions of the Classical swine fever virus (CSFV), Porcine parvovirus (PPV), Porcine circovirus type 2 (PCV2), Japanese encephalitis virus (JEV), Porcine Epidemic Diarrhea Virus (PEDV), Porcine reproductive and respiratory syndrome (PRRSV) and Transmissible gastroenteritis virus (TGEV), all of which showed negative results, demonstrating that the purified antigen was highly specific for the tP72 protein from African swine fever virus.

\section{Sensitivity of the indirect ELISA using tP72}

As shown in Fig. 5, the minimum detection dilution of the indirect ELISA was 1:1600, demonstrating the high sensitivity of the established ELISA.

\section{Discussion}

The sudden outbreak and quick spread of a severe infectious ASF, mainly causes high mortality in pigs. ASFV has huge genomic structure and complex immune evasion mechanisms; there is no effective treatment or vaccine for ASFV (Rowlands et al. 2008; Gallardo et al. 2015; Penrith and Vosloo 2019). The rapid detection and identification of ASFV particles and antibodies were helpful for the prevention and control of African swine fever (Carison et al. 2018; Miao et al. 2019). Quickly detect large numbers of clinical samples using high-sensitivity and specific indirect ELISA methods.

Early studies showed that capsid protein p72 is a major antigen detected in infected pigs and is widely used as a marker of ASFV infection. In the present study, the amplification product was $870 \mathrm{bp}$, which was confirmed by agarose gel electrophoresis (data not shown). We cloned the segment of the p72 protein from amino acids 2 to 291 that showed high antigenic index prediction by DNAMAN software (data not shown). SDS-PAGE analysis showed that the recombinant $\mathrm{tP} 72$ protein was observed with the expected molecular weight of $34 \mathrm{kDa}$. The $\mathrm{tP} 72$ protein accounted for $39 \%$ of the total protein substance, and the purity of was more than $95 \%$ after purification, along with tested by measuring its interaction with his-tag monoclonal antibody and antibody against-ASFV. Western blot results indicated that purified $\mathrm{tP} 72$ was recognized by the anti-His tag monoclonal antibody and polyclonal antibody to ASFV.

Using the purified tP72 protein, an indirect ELISA was developed. We optimized the conditions of the indirect ELISA that was established to be highly sensitive. The optimal concentration of the purified tP72 protein was 3.625 $\mu \mathrm{g} / \mathrm{mL}$ and optimal serum sample dilution was 1:160, and the dilution of the HRP-conjugated secondary antibody was 1:2000. The cutoff value of tP72 indirect ELISA was set at 0.269 , the sample with an $\mathrm{OD}_{450 \mathrm{~nm}}$ value at or above this cutoff was considered positive.

The positive mouse sera against CSFV, PRRSV, PPV, PCV2, JEV, PEDV and TGEV were used to evaluate the specificity of the tP72-based indirect ELISA, the $\mathrm{OD}_{450 \mathrm{~nm}}$ value of the above serum samples were below the critical value. These data showed that the method has great specificity and could distinguish ASFV from other porcine pathogens rapidly.

In this study, we successfully expressed tP72 protein as a recombinant protein in E. coli Rossetta. Additionally, we established an indirect ELISA method based on the tP72 to detect serum antibodies against ASFV. The authors believe that the highly sensitivity and specificity method may be useful for epidemiological surveillance and serological monitoring of ASFV infection.

\section{Conclusion}

In summary, the indirect ELISA established by us could be used for further research of new vaccine development based on this p72 protein of ASFV, and for the establishment of a mouse model to evaluate the efficacy of ASFV vaccine.

\section{Acknowledgements}

This research was supported by major scientific and technological innovation projects of Shandong Province (2019JZZY020606). 


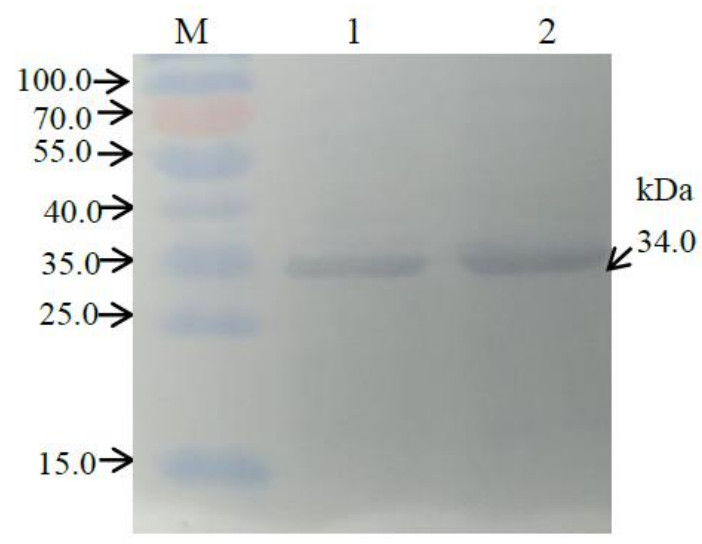

Fig. 3: Western-blotting analysis of ASFV tP72 protein

Lane $\mathrm{M}$ is the protein marker; lane 1 is the result of reaction between anti-his tag monoclonal antibody and recombinant tP72 protein; lane 2, ASFV-positive sera was used to recognize the recombinant $\mathrm{tP} 72$ protein

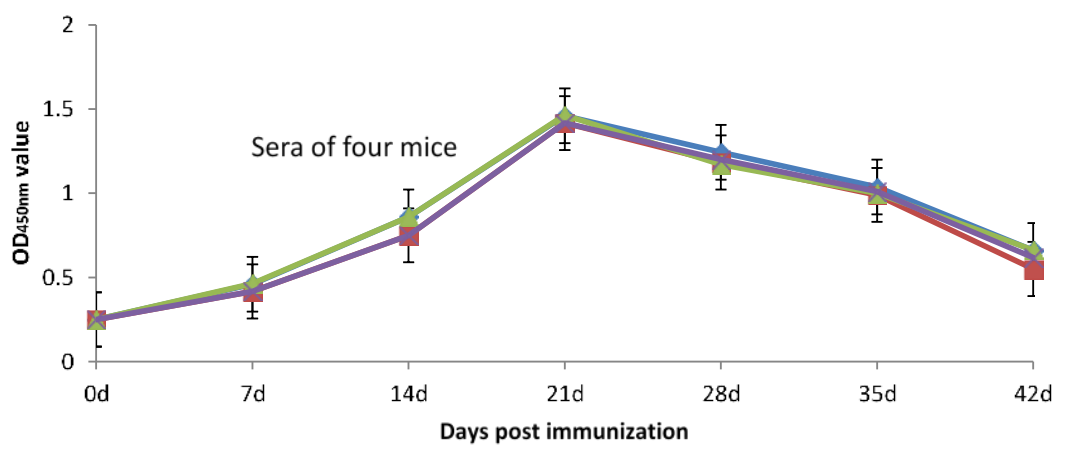

Fig. 4: Serum antibody detection in mice after immunization

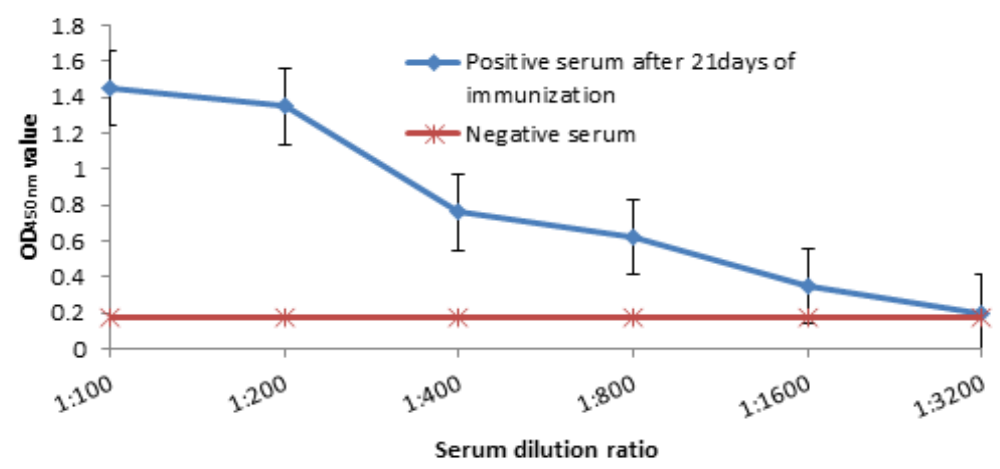

Fig. 5: Sensitivity test of indirect ELISA method based on tP72 protein

\section{Author Contributions}

JLW participated in experiment design and prepared original writing; JLC carried out the experiment and participated in the vector construction; LD participated in protein purification and animal immunity test; XYY and WQM coordinated the study and establishment of ELISA method; ZQS organized the study and revised the manuscript.

\section{References}

Achenbach JE, C Gallardo, E Nieto-Pelegrín, B-Rivera-Arroyo, T Degefa-Negi, M Arias, S Jenberie, DD Mulisa, D Gizaw, E Gelaye, TR Chibssa, A Belaye, A Loitsch, M Forsa, M Yami, A Diallo, A Soler, CE Lamien, JM Sánchez-Vizcaíno (2017). Identification of a new genotype of African swine fever virus in domestic pigs from Ethiopia. Transbound Emerg Dis 64:1393-1404 
Bu RE, J Wang, C DebRoy, J Wu, L Xi, Y Liu, Z Shen (2015). Development of an indirect ELISA for bovine mastitis using Sip protein of Streptococcus agalactiae. Iran J Vet Res 16:283-284

Carison J, L Zani, T Schwaiger, I Nurmoja I, A Viltrop, A Vilem, M Beer, S Blome (2018). Simplifying sampling for African fever surveillance: Assessment of antibody and pathogen detection from bloods swabs. Transbound Emerg Dis 65:165-172

Carolina C, S Gómez-Sebastian, N Moreno, CN María, LK MulumbaMfumu, JQ Carlos, H Livio, MC Eric, J Ferran, ME Jose, B Esther (2013). African swine fever virus serodiagnosis: A general review with a focus on the analyses of African serum samples. Virus Res 173:159-167

Carson M, AL Meredith, DJ Shaw, ES Giotis, DH Lloyd, A Loeffler (2012). Foxes as a potential wildlife reservoir for mecApositive Staphylococci. Vector Borne Zoonot Dis 12:583-587

Costard S, L Mur, J Lubroth, JM Sanchez-Vizcaino, DU Pfeiffer (2013). Epidemiology of African swine fever virus. Virus Res 173:191-197

Crowther J, J Walker (2009). The ELISA Guidebook, $2^{\text {nd }}$ edn. Humana Press, New Jersey, USA

Detray DE (1963). African swine fever. Adv Vet Sci 8:299-333

Galindo I, C Alonso (2017). African swine fever virus: A review. Viruses 9; Article 103

Gallardo C, A Soler, R Nieto, AL Carrascosa, GM De Mia, RP Bishop, C Martins, FO Fasina, E Couacy-Hymman, L Heath, V Pelayo, E Martín, A Simón, R Martín, AR Okurut, I Lekolol, E Okoth, M Arias (2013). Comparative evaluation of novel African swine fever virus (ASF) antibody detection techniques derived from specific ASF viral genotypes with the OIE internationally prescribed serological tests. Vet Microbiol 162:32-43

Gallardo MC, AT Reoyo, J Fernández-Pinero, I Iglesias, MJ Muñoz, ML Arias (2015). African swine fever: A global view of the current challenge. Porcine Health Manage 1; Article 21
Miao F, J Zhang, N Li, T Chen, L Wang, F Zhang, L Mi, J Zhang, S Wang, Y Wang, X Zhou, Y Zhang, M Li, S Zhang, R Hu (2019). Rapid and sensitive recombinase polymerase amplification combined with lateral flow strip for detecting African swine fever virus. Front Microbiol 10; Article 1004

Montgomery RE (1921). On a form of swine fever occurring in British East Africa (KenyaColony). J Compar Pathol Ther 34:159-191

Mulumba-Mfumu LK, JE Achenbach, MR Mauldin, LK Dixon, CG Tshilenge, E Thiry, N Moreno, E Blanco, C Saegerman, CE Lamien, A Diallo, EO Freed (2017). Genetic assessment of African Swine Fever isolates involved in outbreaks in the Democratic Republic of Congo between 2005 and 2012 reveals co-circulation of p72 genotypes I, IX and XIV, including 19 variants. Viruses 9; Article 31

Mur L, M Atzeni, B Martinez-Lopez, F Feliziani, S Rolesu, JM Sanchez-Vizcaino (2016). Thirty-five-year presence of African swine fever in Sardinia: History, evolution and risk factors for disease maintenance. Transbound Emerg Dis 63:165-177

Penrith ML, W Vosloo (2009). Review of African swine fever: Transmission spread and control. J S Afr Vet Assoc 80:58-62

Rowlands RJ, V Michaud, L Heath L, G Hutchings, C Oura, W Vosloo, R Dwarka, T Onashvili, E Albina, LK Dixon (2008). African swine fever virus isolate, Georgia, 2007. Emerg Infect Dis 14:1870-1874

Sanchez-Vizcaino JM, L Mur, B Martinez-Lopez (2012). African swine fever: An epidemiological update. Transbound Emerg Dis 59:27-35

Sastre P, C Gallardo, A Monedero, T Ruiz, M Arias, A Sanz, P Rueda (2016a). Development of a novel lateral flow assay for detection of African swine fever in blood. BMC Vet Res 12; Article 206

Sastre P, T Pérez, S Costa, X Yang, A Räber, S Blome, KV Goller, C Gallardo, I Tapia, J García, A Sanz, P Rueda (2016b). Development of a duplex lateral flow assay for the simultaneous detection of antibodies against African and Classical swine fever viruses. $J$ Vet Diagn Invest 28:543-549 